\title{
Modal Barriers to Controllability in Networks with Linearly-Coupled Homogeneous Subsystems
}

\author{
Mengran Xue and Sandip Roy
}

\begin{abstract}
The controllability of networks comprising homogeneous multi-input multi-output linear subsystems with linear couplings among them is examined, from a modal perspective. The eigenvalues of the network model are classified into two groups: 1) network-invariant modes, which have very high multiplicity regardless of the network's topology; and 2) special-repeat modes, which are repeated for only particular network topologies and have bounded multiplicity. Characterizations of both types of modes are obtained, in part by drawing on decentralizedfixed-mode and generalized-eigenvalue concepts. We demonstrate that network-invariant modes necessarily prevent controllability unless a sufficient fraction of the subsystems are actuated, both in the network as a whole and in any weakly-connected partition. In contrast, the multiplicities of special-repeat modes have no influence on controllability. Our analysis highlights a distinction between built networks where subsystem interfaces may be unavoidable barriers to controllability, and multi-agent systems where protocols can be designed to ensure controllability.
\end{abstract}

\section{INTRODUCTION}

Networks models made up of homogeneous subsystems with linear couplings among them primarily arise in two contexts: 1) the analysis of synchronization phenomena in built networks like the electric power grid or oscillator circuits [1][3]; and 2) the design of control protocols for multi-agent systems [4]-6]. It is recognized that the methods used in the two contexts are very closely connected, with the earlier work on synchronization often guiding the design of controls for multi-agent systems [7]. In both contexts, one recent focus has been to characterize the controllability of these linearly-coupled networks, when inputs can only be applied at a subset of the subsystems [8]-[12]. These controllability analyses have specifically been motivated by questions about: 1) the manipulability of built networks using a sparse set of inputs and 2) the guidance of multi-agent systems from a set of leader agents. A main outcome of these analyses has been to distill controllability for the full model into global (network-level) and subsystem-level controllability conditions, whereupon purely graph-theoretic results can be obtained.

The purpose of this technical note is to examine structural barriers to the controllability of linear-coupled networks that arise due to characteristics of these systems' spectra. The genesis of the study is an omission in the controllability analysis of linearly-coupled networks [8], [13], which was identified in our recent work [9], [14] and also independently in [10]. These

The authors are with the School of Electrical Engineering and Computer Science at Washington State University. This work has been generously supported by National Science Foundation Grants CNS-1545104 and CMMI1635184. Correspondence should be sent to sroy@eecs.wsu.edu. This paper has been submitted to the IEEE Transactions on Automatic Control. Copyright is held by M. Xue and S. Roy. efforts show that global and subsystem-level controllability do not necessarily guarantee controllability of the full model, in the case that the coupled subsystems are general multiinput multi-output (MIMO) devices. Instead, the global and subsystem models can intertwine in a complex way in deciding controllability, because of subtleties in the eigenvector analysis of the models in the case where the full dynamics of the network has repeated eigenvalues. Based on this recognition, here we determine scenarios under which linearly-coupled networks have repeated eigenvalues, and study whether these repeated-eigenvalue scenarios are barriers to controllability. The main outcome of the study is a dichotomy of eigenvalues in linearly-coupled network models with regard to their multiplicity. In particular, eigenvalues of the model can be distinguished as: 1) network-invariant modes, which have very high multiplicity and necessarily prevent controllability unless a high fraction of network nodes are actuated; and 2) specialrepeat modes which have bounded multiplicity, and do not influence controllability. Characterizations are obtained for the two classes of repeated eigenvalues, in part by drawing on fixed-mode notions for decentralized systems and generalizedeigenvalue analysis techniques.

Our study also highlights a distinction in the controllability of built networks as compared to multi-agent systems. Interestingly, although the omission in the controllability analysis has arisen in both contexts [8], [13], there is an important body of work in the multi-agent-systems literature that avoids the issue, and correctly obtains graph-theoretic conditions for controllability [12]. It does so by designing the interaction protocols or input-output couplings among the agents (subsystems) to guarantee controllability. Our study confirms that protocol design can be used generally to eliminate nontrivial network-invariant modes, and hence to reduce controllability to a purely network-graph-based analysis.

The note is organized as follows. The network model is described in Section II. Spectral conditions for controllability are briefly summarized in Section III. In Section IV, the dichotomy in repeated-eigenvalue scenarios is detailed, and its implications on controllability are characterized from an algebraic and graph-theoretic standpoint.

\section{MODEL}

A network comprising identical linear subsystems with linear couplings, which is subject to external actuation at a subset of the subsystems, is considered. Formally, a network with $N$ subsystems, labeled $1, \ldots, N$, is considered. Each subsystem 
$i$ has a state $\mathbf{x}_{i}(t) \in R^{n}$ which evolves in continuous time $\left(t \in R^{+}\right)$. The state of each subsystem $i$ is governed by:

$$
\dot{\mathbf{x}}_{i}=A \mathbf{x}_{i}+B\left(\sum_{j=1}^{N} g_{i j} C \mathbf{x}_{j}+\alpha_{i} \mathbf{u}_{i}\right),
$$

where $A \in R^{n \times n}, B \in R^{n \times m}$, and $C \in R^{m \times n}$ are the common state, input, and output matrices of each subsystem; the scalars $g_{i j}$ indicate the strengths of the couplings between the subsystems; $\mathcal{S}$ is a set of $M$ (distinct) subsystems which can be actuated; $\mathbf{u}_{i} \in R^{m}$ is the input provided to subsystem $i$; and $\alpha_{i}=1$ if $i \in \mathcal{S}$ and $\alpha_{i}=0$ otherwise.

The network's dynamics can be written in assembled form as:

$$
\dot{\mathbf{x}}=\left(I_{N} \otimes A+G \otimes B C\right) \mathbf{x}+(S \otimes B) \mathbf{u}
$$

where $\mathbf{x}=\left[\begin{array}{c}\mathbf{x}_{1} \\ \vdots \\ \mathbf{x}_{N}\end{array}\right], \mathbf{u}$ stacks the input vectors for the subsystems listed in set $\mathcal{S}, G=\left[g_{i j}\right]$, the matrix $S \in R^{N \times M}$ has columns which are $0-1$ indicators vectors of the subsystems listed in $\mathcal{S}, I_{N}$ is an identity matrix with $N$ rows and columns, and $\otimes$ represents the Kronecker product operation. We notice that the triple $(C, A, B)$ specifies the input-output behavior of each subsystem, hence we refer to it as the subsystem model. Meanwhile, the network matrix $G$ and the actuation-location matrix $S$ specify the interaction weights among subsystems and external input locations, respectively, hence we refer to the pair $(G, S)$ as the global model. The subsystem model and the global model together define the full network dynamics (2), which we refer to as the linearly-coupled network.

Our focus in this study is on the controllability of the linearly-coupled network, as specified by the pair $\left(I_{N} \otimes\right.$ $A+G \otimes B C, S \otimes B)$. Many of our analyses are limited to the case that the network matrix $G$ is diagonalizable, which encompasses the symmetric case. We note that many of the network models considered in the literature assume that $G$ has a diffusive structure [1]-[6], [8], [12], which means that $G$ is an essentially-nonnegative matrix (sometimes with the additional requirement that its row sums are 0); however, our analyses here do not depend on this additional structure.

To develop graph-theoretic results, we also find it convenient to define a weighted digraph $\Gamma$ which specifies the topology of interactions among the subsystems. Specifically, $\Gamma$ is defined as a graph on $N$ vertices labeled $1, \ldots, N$, which correspond to the subsystems in the network. An edge is drawn from vertex $j$ to vertex $i(i \neq j)$ if $g_{i j} \neq 0$. The vertices corresponding to the actuated subsystems (i.e., the vertices identified in $\mathcal{S}$ ) are referred to as the actuation locations in the graph.

The linearly-coupled network encompasses models for both built-network dynamics (e.g., power-grid swing dynamics, oscillator synchronization processes) and multi-agent-system coordination. In built-network applications, all network parameters including the interfaces between subsystems are modeled as being fixed, since they capture hardwired interactions. A key distinction in multi-agent systems is that the protocols which interface the network subsystems (agents) are often considered as designable. In particular, the data provided to each agent by the network can be processed in setting the agent's local input. The designability of protocols in multi-agent systems can be captured by modeling the subsystem output matrix $C$ as being designable rather than fixed. In this case, the output matrix $C$ is naturally modeled as taking the form $C=H \widehat{C}$, where $\widehat{C}$ is fixed while $H$ is designable. This formulation captures that subsystem outputs defined by $\widehat{C}$ can be processed in setting local inputs.

Remark: The model considered here is a specialization of those considered in [8]-[11], in that the subsystem couplings and external actuation are both restricted to act through the subsystem input matrix $B$ (mathematically, the range space of the coupling $B C$ is contained within that of the input matrix $B)$. This additional "subsystem" structure is apt for many application domains (see [9] for further discussion), and has a rich modal structure that enables structural characterization of controllability.

\section{Spectral Conditions For Controllability}

Conditions for the controllability of the linearly-coupled network model, which are based on a direct eigenvector analysis of the model, are briefly discussed. These conditions motivate the main study of repeated eigenvalues in the linearly-coupled network model. They also represent a correct treatment of controllability of the model, which addresses the omission noted in [14]. Since similar conditions were presented in a preliminary form in our previous work [9] and/or in the independent study [10], proofs are omitted. To present the results, it is convenient to define the $N$ eigenvalues of the network matrix $G$ as $\lambda_{i}(i=1, \cdots, N)$. We recall that the $N M$ eigenvalues of the full state matrix $I \otimes A+G \otimes B C$ are the union of the eigenvalues of $A+\lambda_{i} B C$ for $i=1, \cdots, N$ [1].

First, a necessary condition for controllability is presented in the following lemma:

Lemma 1: The linearly-coupled network, as defined by the pair $(I \otimes A+G \otimes B C, S \otimes B)$, is controllable only if 1$)$ the pair $(G, S)$ is controllable and 2$)$ the pairs $\left(A+\lambda_{i} B C, B\right)$ are controllable for $i=1, \ldots, N$.

This necessary condition can be further simplified into separate conditions on the global and subsystem models, via a simple application of the Hautus lemma:

Corollary 1: The linearly-coupled network, as defined by the pair $(I \otimes A+G \otimes B C, S \otimes B)$, is controllable only if 1) the global model is controllable (i.e., the pair $(G, S)$ is controllable) and 2) the subsystem model is controllable (i.e., the pair $(A, B)$ is controllable).

There has been inclarity about whether the condition in Lemma 1 is also sufficient, as noted in [9], [10], [14]. Essentially, the reason why the condition is not sufficient is that the matrices $A+\lambda_{i} B C, i=1, \ldots, N$, may share eigenvalues across them. These eigenvalues are repeated eigenvalues of the full network model (2), whose eigenspaces may contain 
eigenvectors other than ones that are Kronecker products of the global- and subsystem-level- eigenvectors. Hence, it is possible that the PBH test for controllability may fail, even when the global and subsystem models are controllable. We also notice that the simplification in Corollary 1 depends on the network having a "subsystem" structure, rather than arbitrary couplings among its components.

In the case where the network matrix $G$ is diagonalizable, a necessary and sufficient condition for controllability can be obtained by enumerating the eigenvectors associated with each repeated eigenvalue of the full network model (2). The condition requires some additional notation. We refer to the left eigenvectors of $G$ associated with each eigenvalue $\lambda_{j}$, $j=1, \ldots, N$, as $\mathbf{v}_{j}^{T}$. Further, for each distinct eigenvalue $\mu_{i}$, $i=1, \ldots, z$, of the full network dynamics (2), the geometric multiplicity of the eigenvalue in the matrix $A+\lambda_{j} B C$ is denoted as $\beta_{j}\left(\mu_{i}\right)$. The corresponding left eigenvectors of $A+\lambda_{j} B C$ (if there are any) are denoted as $\mathbf{w}_{j l}^{T}$, where $l=1, \ldots, \beta_{j}\left(\mu_{i}\right)$. Here is the necessary and sufficient condition:

Lemma 2: Assume that the linearly-coupled network (2) has a diagonalizable network matrix $G$. The network, as defined by the pair $(I \otimes A+G \otimes B C, S \otimes B)$, is controllable if and only if the following condition holds for each $i=1, \ldots, z$ : $\sum_{j=1}^{N} \sum_{l=1}^{\beta_{j}\left(\mu_{i}\right)} a_{j, l}\left(\mathbf{v}_{j}^{T} S\right) \otimes\left(\mathbf{w}_{j l}^{T} B\right) \neq 0$ for any real scalar coefficients $a_{j, l}$ that are not all identically 0 .

The necessary and sufficient condition in Lemma 2 is important as a complete formal treatment of controllability of the linearly-coupled network [9], [10]. However, it is sometimes difficult to apply directly to obtain graph-theoretic or structural results on controllability, because the roles of the global and subsystem models are intertwined in a complicated way. Noting that the intertwining of the global and subsystem conditions is tied to the presence/absence of repeated eigenvalues across the matrices $A+\lambda_{i} B C$, simpler sufficient or necessary conditions for controllability can be obtained based on eigenvalue multiplicities. Two useful results of this sort are formalized in the following lemma:

Lemma 3: Consider the linearly-coupled network, as defined by the pair $(I \otimes A+G \otimes B C, S \otimes B)$, for diagonalizable $G$. The model is controllable if the following two conditions hold: 1) the global model $(G, S)$ is controllable; 2$)$ no two matrices $A+\lambda_{i} B C$ and $A+\lambda_{j} B C(i=1, \ldots, N, j=1, \ldots, N)$ such that $\lambda_{i} \neq \lambda_{j}$ share eigenvalues. Meanwhile, the linearlycoupled network model is not controllable if $M m<P(\mu)$ for any complex number $\mu$, where $P(\mu)$ is the sum of the geometric multiplicities of an eigenvalue $\mu$ across matrices $A+\lambda_{i} B C$.

The lemma shows that controllability reduces to a global (or graph- based) condition, if the matrices $A+\lambda_{i} B C$ corresponding to distinct $\lambda_{i}$ do not share eigenvalues. We note here that the subsystem's controllability is necessary (but not sufficient) for the matrices $A+\lambda_{i} B C$ to not share eigenvalues. On the other hand, controllability is necessarily lost if an eigenvalue recurs in a sufficient number of blocks $A+\lambda_{i} B C$ as compared to the number of inputs. We note that these conditions may not be tight, as controllability depends on the specific structure of the eigenspaces associated with the repeated eigenvalues. However, the result focuses attention on the close relationship between repeated eigenvalues across the matrices $A+\lambda_{i} B C$ and controllability, and provides a means to assess controllability solely via an eigenvalue analysis.

Remark: The controllability analysis become more sophisticated if $G$ is a defective matrix, because the linearly-coupled network's state matrix $I_{N} \otimes A+G \otimes B C$ may have eigenvectors that neither Kronecker products of global and subsystem-level eigenvectors, nor combinations thereof. We refer the reader to [15], [16] for an eigenvector analysis for Kronecker products of defective matrices, which is a starting point for a treatment of the linearly-coupled network with defective $G$.

\section{A Dichotomy of RepeAted-Eigenvalue SCENARIOS}

In this section, we study conditions under which matrices of the form $A+\lambda B C$ may share eigenvalues for two distinct complex scalars $\lambda$. The main outcome of the analysis is a dichotomy of scenarios:

1) In some cases, matrices $A+\lambda B C$ have eigenvalues that are fixed over the field of complex $\lambda$, i.e. all matrices of this form have shared eigenvalue(s). We refer to such eigenvalues as network-invariant modes.

2) Alternately, in other cases, the matrices $A+\lambda B C$ only share eigenvalues if the scalars $\lambda$ are chosen from particular finite sets with bounded cardinality. We refer to these eigenvalues as special-repeat modes.

These scenarios are differentiated and characterized in the following development, via formal analyses and examples. Also, the implications of the scenarios on the controllability of the linear-network model are determined. A main finding is that network-invariant modes place severe restrictions on controllability of the linearly-coupled network, while specialrepeat modes cannot modulate controllability. This analysis also differentiates controllability of built networks as compared to designable multi-agent systems.

We note that there has been an intense research effort to characterize the spectra of matrices of the form $A+\lambda B C$ (or $A+\lambda D$ ) over the field of complex $\lambda$, primarily to analyze the stability of network synchronization and multiagent system dynamics [1]-[6]. The stability analysis is often encoded using a master stability function, which identifies the region of values $\lambda$ in the complex plane for which Hurwitz stability is achieved. The possible shapes of the master stability region have been studied extensively [17]. To the best of our knowledge, however, scenarios where the matrices $A+\lambda B C$ share eigenvalues at multiple points in the complex plane have not been determined. Our study approaches this question, in part by noting connections to decentralized-control and generalized-eigenvalue notions.

\section{A. Scenarios with Network-Invariant Modes}

We first consider the possibility that the matrices $A+\lambda B C$ may have eigenvalues that remain fixed over the entire field 
of complex $\lambda$. Formally, let us define an eigenvalue that is invariant over the field of complex $\lambda$ as follows:

Definition 1: A complex number $\mu$ is called a networkinvariant mode of the linearly-coupled network (2), if $\mu$ is an eigenvalue of $A+\lambda B C$ for any complex $\lambda$.

We first delineate scenarios under which the linearly-coupled network has network-invariant modes. We then study the implications of network-invariant modes on the controllability of the linearly-coupled network.

First, it is apparent that any eigenvalue of $A$ which is an uncontrollable or unobservable mode of the subsystem model is a network-invariant mode. This is because an uncontrollable or unobservable eigenvalue is invariant to any feedback, in the sense that it is an eigenvalue of $A+B K C$ for any complex matrix $K$, including $K=\lambda I$. It is natural to ask whether the uncontrollable and unobservable eigenvalues of the subsystem model are the only network-invariant modes. If this is the case, then uncontrollability for a generic network matrix may only arise in the degenerate case that the subsystem model is uncontrollable or unobservable. However, the following example illustrates that the linearly-coupled network may have network-invariant modes even if the subsystem model is controllable and observable, and indeed controllability of the linearly-coupled network may be lost:

Example 1: Consider a linear-coupled network with: $A=$ $\left[\begin{array}{lll}0 & 0 & 0 \\ 0 & 0 & 1 \\ 1 & 0 & 1\end{array}\right], B=\left[\begin{array}{ll}1 & 0 \\ 0 & 1 \\ 0 & 0\end{array}\right], C=\left[\begin{array}{lll}1 & 0 & 0 \\ 0 & 2 & 0\end{array}\right], G=$ $\left[\begin{array}{ccc}2 & -2 & 0 \\ -2 & 4 & -2 \\ 0 & -2 & 2\end{array}\right]$, and $S=\left[\begin{array}{l}1 \\ 0 \\ 0\end{array}\right]$. For this model, the local subsystem is immediately seen to be both observable and controllable. However, the matrix $A+\lambda B C$ is equal to $\left[\begin{array}{ccc}\lambda & 0 & 0 \\ 0 & 2 \lambda & 1 \\ 1 & 0 & 1\end{array}\right]$. Hence, $A+\lambda B C$ has a network-invariant mode at $\mu=1$. Noting that $G$ is diagonalizable, it follows immediately the state matrix of the linearly-coupled network model has an eigenvalue $\mu=1$ with geometric multiplicity 3 (one eigenvalue at 1 corresponding to each of the blocks $A+\lambda_{i} B C$, where $\lambda_{i}=0,2,6$ are the eigenvalues of $G$ for the example). Meanwhile, the model has only two inputs (both applied at subsystem 1), hence it cannot be controllable.

Example 1 shows that network-invariant modes may be present even when the local subsystem does not have unobservable/uncontrollable modes, because matrices of the form $A+\lambda B C$ represent a structured feedback as compared to arbitrary matrices of the form $A+B K C$. The example is a starting point point toward a more general understanding of structures that lead to network-invariant modes. In particular, the local subsystem in the example can be seen to have a decentralized fixed mode [18] if each input and measurement is considered as a separate feedback channel, even though it is centrally observable and controllable. Indeed, in general, decentralized fixed modes in the local subsystem are necessarily network-invariant modes of the linearly-coupled network. To formalize this concept, we apply the definition of a decentralized fixed mode, which was initially developed in the Wang and Davison's seminal work on decentralized control [18], to the local-subsystem model.

Definition 2: The complex number $\mu$ is said to be a decentralized fixed mode of the local-subsystem model $(C, A, B)$, if $\mu$ is an eigenvalue of $A+B K C$ for any real diagonal matrix $K$.

The decentralized fixed modes of the local subsystem model are a subset of the network-invariant modes, as formalized in the following lemma:

Lemma 4: If $\mu$ is a decentralized fixed mode of the local subsystem model, it is also a network-invariant mode of the linearly-coupled network.

Proof: Since $\mu$ is a decentralized fixed mode of the local subsystem model, $\operatorname{det}(s I-(A+B K C))=0$ has a solution at $s=\mu$ for all real diagonal $K$. Choosing $K=\lambda I$, we immediately recover that $\operatorname{det}(s I-(A+\lambda B C))=0$ has a solution at $s=\mu$ for all real $\lambda$, i.e. $\operatorname{det}(\mu I-(A+\lambda B C))=0$ for all real $\lambda$.

Now consider $\operatorname{det}(\mu I-(A+\lambda B C))$ over the field of complex $\lambda$. The determinant is a polynomial in $\lambda$ with degree equal to rank $B C$. It follows that the equation $\operatorname{det}(\mu I-(A+$ $\lambda B C))=0$ either holds for a finite set of $\lambda$ in the complex plane, or holds for all $\lambda$. Since the equation holds for all real $\lambda$, it thus must hold for all $\lambda$. We have thus shown that $\mu$ is an eigenvalue of $A+\lambda B C$ for all complex $\lambda$, i.e. it is a network-invariant mode of the linearly-coupled model.

Numerous algorithmic as well as structural methods for understanding decentralized fixed modes have been developed in the literature [18]-[20]. Per Lemma 4, these methods can be applied to characterize local subsystem models that lead to network-invariant modes.

Remark: An immediate consequence of Lemma 4 is that the ordering of the subsystem input and output channels may influence the presence of network-invariant modes, and hence controllability of the network. For example, in Example 1, the full network model (2) becomes controllable when the two columns of $B$ are switched (i.e., $B=\left[\begin{array}{ll}0 & 1 \\ 1 & 0 \\ 0 & 0\end{array}\right]$ ).

The linearly-coupled network may be expected to have network-invariant modes other than the subsystem decentralized fixed modes, since the matrix $A+\lambda B C$ has an even more restricted form than allowed by application of decentralized feedback (specifically, the decentralized feedback gains applied to each channel must be identical). The following example shows that the model may have network-invariant modes even when it does not have decentralized fixed modes:

Example 2: Consider a linearly-coupled network model with: $A=\left[\begin{array}{ccc}0 & 0 & 1 \\ 0 & 0 & 1 \\ 1 & -1 & 1\end{array}\right], B=\left[\begin{array}{ll}1 & 0 \\ 0 & 1 \\ 0 & 0\end{array}\right], C=\left[\begin{array}{lll}1 & 0 & 0 \\ 0 & 1 & 0\end{array}\right]$, For this model, it can be verified that the local subsystem model does not have decentralized fixed modes, by checking the eigenvalues of $A+B K C$ for two randomly-selected 
diagonal $K$ (see [18]). However, the matrix $A+\lambda B C$ is equal to $\left[\begin{array}{ccc}\lambda & 0 & 1 \\ 0 & \lambda & 1 \\ 1 & -1 & 1\end{array}\right]$. For any complex $\lambda$, this matrix has an eigenvalue at $\mu=1$ with corresponding right eigenvector equal to $\left[\begin{array}{c}1 \\ 1 \\ 1-\lambda\end{array}\right]$. Thus, the linear-network model has a networkinvariant mode at $\mu=1$ even though the subsystem model does not have decentralized fixed modes.

The above development has shown that network-invariant modes of the linear network model are a superset of the decentralized fixed modes of the subsystem model (which are themselves a superset of the unobservable and uncontrollable modes of the subsystem model).

Remark: Scaling of the subsystem inputs or outputs may also influence whether network-invariant modes are present, and hence may alter the controllability of the network. For instance, in Example 2, if the $B$ matrix is changed to $B=$ $\left[\begin{array}{ll}1 & 0 \\ 0 & 2 \\ 0 & 0\end{array}\right]$, then the network-invariant mode is eliminated.

Remark: In the special case that the subsystem model has a single input and single output, the set of network-invariant modes are trivially seen to be precisely the unobservable and uncontrollable modes.

The presence of network-invariant modes necessarily prevents controllability of the linearly-coupled network, under broad conditions on the global model $(G, S)$. In particular, if a network-invariant mode is present, matrices $A+\lambda_{i} B C$ share the mode for all $\lambda_{i}$ of $G$. Hence, this mode has large geometric multiplicity in the full linearly-coupled model (2) provided that $G$ is diagonalizable. Thus, the linearly-coupled network model can be controllable only if actuation is provided at a sufficient number of network channels, regardless of the network matrix $G$. This concept is formalized in the following lemma:

Lemma 5: Consider a linearly-coupled network model (2) with diagonalizable network matrix $G$, which has a networkinvariant mode. The model is controllable only if the number of actuation locations satisfies $M \geq\left\lceil\frac{N}{m}\right\rceil$.

Proof: For diagonalizable $G$, the geometric multiplicity of the network-invariant mode is at least $N$. Thus, from Lemma 3. controllability requires that $M m \geq N$. The result follows.

Lemma 5 shows that controllability requires actuation at a specified fraction of the subsystems (at least), whenever network-invariant modes are present. This is true regardless of the graph $\Gamma$ of the linearly-coupled network. Controllability cannot be achieved through design of the network topology or selection of particular actuation locations, if the number of actuation locations is insufficient.

Lemma 5 can be further refined to show that any weaklyconnected partition of the network must have a sufficient number of actuated nodes for controllability:

Theorem 1: Consider a linearly-coupled network with diagonalizable network matrix $G$ which has a network-invariant mode. Consider any subset $\mathcal{T}$ of the network's subsystems (correspondingly, vertices in the graph $\Gamma$ ). The linearlycoupled network is controllable only if $\widehat{M} \geq\left\lceil\frac{\widehat{N}}{m}\right\rceil-b$, where $\widehat{N}$ is the total number of vertices within $\mathcal{T}, \widehat{M}$ is the number of actuation locations within $\mathcal{T}$, and $b$ is the number of vertices in $\mathcal{T}$ which are not actuation locations but have an incoming edge from vertices outside $\mathcal{T}$ in $\Gamma$.

Proof: Without loss of generality, we assume that $\mathcal{T}$ contains subsystems $1, \ldots, \widehat{N}$. The dynamics of the subsystems within within $\mathcal{T}$ can be expressed as

$$
\dot{\hat{\mathbf{x}}}=\left(I_{\widehat{N}} \otimes A+\widehat{G} \otimes B C\right) \widehat{\mathbf{x}}+\widehat{S} \otimes B \widehat{\mathbf{u}} .
$$

In Equation 3, $I_{\widehat{N}}$ is an identity matrix with $\widehat{N}$ rows; $\widehat{G}$ is the principal submatrix of $G$ containing the first $\widehat{N}$ rows and columns; and $\widehat{S}$ is a matrix with $\widehat{N}$ rows and $\widehat{M}+b$ columns, where the columns are $0-1$ indicator vector of the $\widehat{M}$ actuation locations and the $z$ additional vertices which have incoming edges from vertices outside $\mathcal{T}$. The vector $\widehat{\mathbf{u}}$ concatenates vectors $\widehat{\mathbf{u}}_{i}$ corresponding to the $\widehat{M}+z$ subsystems indicated by $\widehat{S}$, where each $\widehat{\mathbf{u}}_{i}$ is the sum of the external input signal $\mathbf{u}_{i}$ at the subsystem (if there is an input $\mathbf{u}_{i}$ at this subsystem) and a signal projected from the network outside $\mathcal{T}$ (specifically, $\left.\sum_{j \notin \mathcal{T}} g_{i j} C \mathbf{x}_{j}\right)$.

Controllability of the linearly-coupled network requires controllability of the system (3), where $\widehat{\mathbf{u}}$ is considered as an input signal in (3). This is because, if system (3) is not controllable, there is at least one state $\widehat{\mathrm{x}}=\overline{\hat{\mathrm{x}}}$ that cannot be achieved via the applied input $\widehat{\mathbf{u}}$. Thus, in this case, the linearly-coupled network model (2) also necessarily cannot be driven to an arbitrary state. Thus, controllability of (3) is necessary for controllability of (2).

However, the system (3) is a modified linearly-coupled network model, which has network matrix $\widehat{G}$ rather than $G$, and actuation-location matrix $\widehat{S}$ rather than $S$. This modified model is immediately seen to have the same network-invariant mode as the original network. Thus, from Lemma 5 , it follows that $\widehat{M}+z \geq\left\lceil\frac{\widehat{N}}{m}\right\rceil$, and the theorem statement follows.

Theorem 11 shows that linearly-coupled networks with network-invariant modes must have a sufficient density of actuation in all parts of the network for controllability. That is, not only is actuation required at a certain fraction of the subsystems overall, but each partition of the network requires actuation at a sufficient number of contained subsystems.

For a broad subclass of linear network models with networkinvariant modes, the requirement for controllability is even more stringent. In particular, network-invariant modes often have the characteristic that the eigenvector of $A+\lambda B C$ associated with the mode has the same projection on the subsystem input matrix for every $\lambda$. Formally, let us define $\overline{\mathbf{w}}(\lambda)^{T}$ to be the left eigenvector of $A+\lambda B C$ associated with a particular network-invariant mode $\mu$. We say that the networkinvariant mode $\mu$ is projection-fixed, if the product $\overline{\mathbf{w}}(\lambda)^{T} B$ is identical for all $\lambda$, to within a multiplicative factor (i.e. $\overline{\mathbf{w}}(\lambda)^{T} B=\alpha(\lambda) \mathbf{p}$, where $\mathbf{p}$ is a common row vector for all $\lambda$ and $\alpha(\lambda)$ is a scalar multiplicative factor that depends on $\lambda$ ). 
The following lemma shows that a linear network model with subsystem-projection-fixed network-invariant modes can only be controllable if actuation is provided at all network nodes:

Lemma 6: Consider a linearly-coupled network (2) with diagonalizable $G$. If the model has any network-invariant modes which are projection-fixed, then the linear network model is controllable only if actuation is provided at all network nodes $(M=N)$.

Proof: We apply Lemma 2 to the projection-fixed networkinvariant mode, say $\mu$, to show necessity. In particular, the linearly-coupled network is controllable only if: $\sum_{j=1}^{N} \sum_{l=1}^{\beta_{j}(\mu)} \alpha_{j, l}\left(\mathbf{v}_{j}^{T} S\right) \otimes\left(\mathbf{w}_{j l}^{T} B\right) \neq 0$ for any real scalar coefficients $\alpha_{j, l}$ that are not all identically 0 . Since $\mu$ is a network-invariant mode, the mode's multiplicity $\beta_{j}(\mu)$ in each block $A+\lambda_{j} B C$ is necessarily at least 1 . Meanwhile, since the mode is projection-fixed, $\mathbf{w}_{j l}^{T} B$ is proportional to some row vector $\mathbf{p}$ for all $j$ and $l$. Thus, the necessary condition for controllability can be simplified to $\sum_{j=1}^{N} \alpha_{j}\left(\mathbf{v}_{j}^{T} S\right) \otimes \mathbf{p} \neq 0$ for all $\alpha_{j}$. However, this is only possible of $S$ has $N$ independent columns, which necessitates that $M=N$.

It is easy to check that the network-invariant modes in Examples 1 and 2 are both projection-fixed, and hence actuation at all network locations is necessary for controllability. Although many network-invariant modes are projection-fixed, not all are. The following is an example of a linearly-coupled network with a network-invariant mode that is not projectionfixed:

Example 3: Consider a linear network model with: $A=$ $\left[\begin{array}{ccc}0.6 & 0.4 & 0 \\ 0.2 & 0.7 & 0.1 \\ 0 & 0.2 & 0.8\end{array}\right], B=\left[\begin{array}{llll}1 & 0 & 1 & 0 \\ 0 & 1 & 0 & 1 \\ 0 & 0 & 0 & 0\end{array}\right], C=\left[\begin{array}{ccc}1 & 0 & 0 \\ 0 & -1 & 0 \\ 0 & -1 & 0 \\ 1 & 0 & 0\end{array}\right]$, $G=\left[\begin{array}{ccc}1 & -1 & 0 \\ -1 & 2 & -1 \\ 0 & -1 & 1\end{array}\right]$, and $\mathcal{S}=\{1,2\}$. It can be checked that the model has a network-invariant mode at $\mu=1$ that is not projection-fixed. The linear network model is found to be controllable, even though actuation has only been provided at two of the three subsystems.

The above analysis shows that linearly-coupled networks may have network-invariant modes, which can place essential limits on controllability regardless of the topology of the network. Thus, controllability of built networks (i.e., linearlycoupled networks with fixed interfaces) requires additional conditions on the subsystems beyond local controllability and observability. For instance, (centralized) controllability of the linearly-coupled network model is tied to decentralized controllability of the subsystem model.

These barriers to controllability seem surprising at first glance, since the controllability of multi-agent system models has been distilled to a purely graph-theoretic condition in the literature [12]. This difference arises because multi-agent system models allow design of the interaction protocols among the agents, in contrast with built networks. The difference can be explained within our framework by considering the linearly-coupled network model with designable $C$ matrix, which captures the multi-agent-system context:

Lemma 7: Consider a linearly-coupled network model for which the subsystem's output matrix takes the form $C=H \widehat{C}$, where $H$ is designable. Provided that the subsystem model $(\widehat{C}, A, B)$ is observable and controllable, the matrix $H$ can be designed so that the linear network model has no networkinvariant modes.

Proof: Consider the eigenvalues of $A+\lambda B C=A+\lambda B H \widehat{C}$ for $\lambda=0$ and $\lambda=1$. For $\lambda=0$, the eigenvalues are those of $A$. For $\lambda=1$, the eigenvalues are those of $A+B H \widehat{C}$. Since $(\widehat{C}, A, B)$ is observable and controllable, $H$ can be chosen so that the eigenvalues of $A+B H \widehat{C}$ all differ from those of the matrix $A$ (i.e., a feedback can be applied to move all eigenvalues). For such a choice of $H, A+\lambda B C$ does not share any eigenvalues for $\lambda=0$ and $\lambda=1$, hence the model has no network-invariant modes.

The lemma formalizes that multi-agent-system protocols can always be designed to avoid network-invariant modes, by choosing any matrix $H$ such that the eigenvalues of $A+B H C$ differ from those of $A$.

\section{B. Scenarios with Special-Repeat Modes}

Even if the linear network model does not have networkinvariant modes, the blocks $A+\lambda_{i} B C$ may share eigenvalues for particular $\lambda_{i}$. These repeated eigenvalues putatively may also interfere with controllability, since their associated eigenvectors across the blocks may lie in the null space of the input matrix, per Lemma 2 To gain an understanding of whether the blocks $A+\lambda_{i} B C$ can share eigenvalues for special choices of $\lambda_{i}$, we consider solving for the $\lambda$ for which $A+\lambda B C$ has a particular eigenvalue $\mu$. For such $\lambda$, the equation $\mathbf{w}^{T}(A+\lambda B C)=\mu \mathbf{w}^{T}$ must be satisfied for some $\mathbf{w}^{T}$. Rearranging, the equation can be written as:

$$
\mathbf{w}^{T}(A-\mu I)=\lambda \mathbf{w}^{T}(-B C)
$$

Equation 4 clarifies that finding the $\lambda$ values that yield a particular eigenvalue $\mu$ corresponds to solving a generalized eigenvalue problem for the pair $(A-\mu I,-B C)$. From standard results on generalized eigenvalue problems [21], [22], only the following scenarios are possible:

- The generalized eigenvalue problem is degenerate, in the sense that it has a solution for every $\lambda$ (i.e., there is vector in the left-null-space of $A+\lambda B C-\mu I$ from every $\lambda$ ). This corresponds to the case that that the linear network model has network-invariant modes.

- Alternately, the generalized eigenvalue problem has a solution for a finite set of $\lambda$, with the number of distinct solutions less than or equal to the rank of the matrix $B C$. We refer to eigenvalues $\mu$ that only repeat for a finite set of $\lambda$ as special-repeat modes, and refer to the corresponding set of distinct $\lambda$ values as the networkrepeat set for $\mu($ or $N R(\mu))$.

The generalized-eigenvalue formulation leads to the following characterization of the special-repeat modes and networkrepeat sets: 
Lemma 8: Consider an eigenvalue $\mu$ that is a specialrepeat mode of a linearly-coupled network (2). The maximum number of distinct $\lambda$ for which $A+\lambda B C$ has eigenvalue $\mu$ (i.e., the maximum possible size for the network-repeat set $N R(\mu)$ ) is at most $\operatorname{rank}(B C)$. Further, consider the set of vectors $\mathbf{w}^{T}$ for which $\mathbf{w}^{T}(A+\lambda B C)=\mu \mathbf{w}^{T}$, for $\lambda \in N R(\mu)$. There are $r \leq \operatorname{rank}(B C)$ such vectors, say $\mathbf{w}_{1}^{T}, \ldots, \mathbf{w}_{r}^{T}$, which are linearly independent. Further, the projections of these vectors into the matrix $B$ are linearly independent, i.e. $\mathbf{w}_{1}^{T} B, \ldots, \mathbf{w}_{r}^{T} B$ are linearly independent.

Proof: The number of the solutions $\lambda$, and the fact that there are at most $\operatorname{rank}(B C)$ vectors that satisfy $\mathbf{w}^{T}(A+\lambda B C)=$ $\mu \mathbf{w}^{T}$, are standard results on generalized eigenvalues [21].

It remains to prove that the projections of the generalized eigenvectors $\mathbf{w}_{1}^{T} B, \ldots, \mathbf{w}_{r}^{T} B$ are linearly independent. We will prove the result by contradiction. If the vectors are not linearly independent, then there exist $\gamma_{1}, \ldots, \gamma_{r}$ which are not identically zero such that $\sum_{i=1}^{r} \gamma_{i} \mathbf{w}_{i}^{T} B=0$. Notice that this is only possible for $r \geq 2$. To continue, we scale and sum the equations $\mathbf{w}_{i}^{T}\left(A+\lambda_{i} \bar{B} C\right)=\mu \mathbf{w}_{i}^{T}$ for $i=1, \ldots, r$, where $\lambda_{i}$ are the corresponding generalized eigenvalues in $N R(\mu)$. In particular, we can get:

$$
\sum_{i=1}^{r} \frac{\gamma_{i}+c_{i}}{\lambda_{i}} \mathbf{w}_{i}^{T}\left(A+\lambda_{i} B C\right)=\mu \sum_{i=1}^{r} \frac{\gamma_{i}+c_{i}}{\lambda_{i}} \mathbf{w}_{i}^{T},
$$

where $c_{1}, \ldots, c_{r}$ are scalars. Multiplying out the left side of (5) and then using $\sum_{i=1}^{r} \gamma_{i} \mathbf{w}_{i}^{T} B=0$, we get:

$$
\sum_{i=1}^{r} \frac{\gamma_{i}+c_{i}}{\lambda_{i}} \mathbf{w}_{i}^{T} A+\sum_{i=1}^{r} c_{i} \mathbf{w}_{i}^{T} B C=\mu \sum_{i=1}^{r} \frac{\gamma_{i}+c_{i}}{\lambda_{i}} \mathbf{w}_{i}^{T},
$$

Choosing $c_{i}=\frac{h \gamma_{i}}{\lambda_{i}-h}$ for any scalar $h \neq \lambda_{i}$, one recovers that:

$$
\sum_{i=1}^{r} c_{i} \mathbf{w}_{i}^{T}(A+h B C)=\mu \sum_{i=1}^{r} c_{i} \mathbf{w}_{i}^{T} .
$$

Thus, $\mu$ is seen to be an eigenvalue of $A+h B C$ for any scalar $h$, with corresponding eigenvector $\sum_{i=1}^{r} c_{i} \mathbf{w}_{i}^{T}$. This contradicts the fact that $\mu$ is a special-repeat mode, since it is an eigenvalue for a continuum of $h$ rather than a finite set. Thus, the result is proved by contradiction. $\square$

Lemma 8 indicates the matrices $A+\lambda_{i} B C$ may have repeated eigenvalues across them, however the total multiplicity is limited by $\operatorname{rank}(B C)$, and further the corresponding eigenvectors' projections on $B$ are linearly independent. In fact, these limitations on special-repeat modes' eigenspaces have implications on controllability of the linearly-coupled network model, as is formalized in the following theorem:

Theorem 2: Consider a linearly-coupled network (2) with diagonalizable $G$, Further, assume that all eigenvalues of the model are special-repeat modes (i.e., the model has no network-invariant modes). Then, if the global model is controllable, the linearly-coupled network model is controllable.

Proof: From Lemma 2, the linearly-coupled network model is controllable if $\sum_{j=1}^{N} \sum_{l=1}^{\beta_{j}(\mu)} \alpha_{j, l}\left(\mathbf{v}_{j}^{T} S\right) \otimes\left(\mathbf{w}_{j l}^{T} B\right) \neq 0$ for all $\alpha_{j, l}$ which are not identically 0 , for each distinct eigenvalue $\mu$ of the linearly-coupled network model. Consider a particular eigenvalue $\mu$, which by assumption is a special-repeat mode. Consider the case that $\mu$ is an eigenvalue of $A+\lambda_{i} B C$ for $g$ distinct eigenvalues $\lambda_{i}$ of $G$, where $g \leq \operatorname{rank}(B C)$ from Lemma 8, let us refer to these eigenvalues of $G$ a $\lambda_{1}, \ldots, \lambda_{g}$ without loss of generality. (We notice that these eigenvalues of $G$ also may also recur in other blocks $\left.A+\lambda_{i} B C\right)$. In this notation, the controllability condition can be written as: $\sum_{j=1}^{g} \sum_{l=1}^{\beta_{j}(\mu)} \alpha_{j, l}\left(\mathbf{v}_{j}^{T} S\right) \otimes\left(\mathbf{w}_{j l}^{T} B\right)$, where $\mathbf{v}_{j}^{T}$ may be any eigenvector associated with the eigenvalue $\lambda_{j}$. From Lemma 8 , we notice that $\mathbf{w}_{j l}^{T} B$ for each pair $(j, l)$ is linearly independent. Thus, the eigenvalue $\mu$ is controllable if $\mathbf{v}_{j}^{T} S \neq 0$ for all $j$. However, this is guaranteed from controllability of the global model. The argument can be repeated for each distinct eigenvalue $\mu$ of the linearly-coupled network model, hence controllability is shown.

Theorem 2 demonstrates that special-repeat eigenvalues are harmless, in the sense that they cannot prevent controllability of the linear network model.

Remark: In contrast with the result presented here, specialrepeat-type modes (i.e., eigenvalues of $A+\lambda B C$ that repeat only for finite sets of $\lambda$ ) can block controllability for the broader class of network models considered in [8]-[10]. Indeed, the counterexample shown in the comment [14] arises from a special-repeat-type mode. The reason for the difference is that the linear couplings and the subsystem input matrix do not have the same range space in these models (i.e., the coupling matrix is $D \neq B C$ ). In this broader circumstance, special-repeat modes can result in uncontrollability, which means that particular choices of the network matrix may lead to uncontrollability while other choices allow control.

\section{Discussion}

Our analysis has shown that the eigenvalues of the linear network model can be categorized into two types, which we call network-invariant modes and special-repeat modes. The two types of modes, and their implications on network controllability, have been characterized above. A key outcome of the analysis is that controllability of the linear-network model is solely tied to the presence or absence of network-invariant modes, rather than special-repeat modes. This characterization of controllability has several interesting implications:

- A linearly-coupled network model without networkinvariant modes (with diagonalizable $G$ ) is controllable only if the global model $(S, B)$ is controllable. Thus, controllability can be distilled to a condition on only the network topology and input locations in this case. The wide range of graph-theoretic results on the controllability of scalar diffusive/consensus processes can be brought to bear (e.g. [23]), if the topology matrix $G$ has a diffusive structure.

- If the linearly-coupled network model has networkinvariant modes, then controllability is lost regardless of the network's graph topology, unless actuation is provided at a sufficient fraction of nodes. If these network-invariant 
modes are projection-fixed, then controllability is lost unless actuation is provided at all nodes in the network.

- If the subsystem model is SISO, then the linearly-coupled network model has network-invariant modes if and only if the subsystem is either unobservable or uncontrollable. Thus, the linear network model is controllable if 1) the global model is controllable, and 2) the subsystem model is controllable and observable.

- The controllability of built networks differs from that of multi-agent systems, because of the design freedom available in multi-agent-system control. In particular, protocols in multi-agent systems can be designed to avoid network-invariant modes, which means that they can be designed so that controllability reduces entirely to a graph-level condition. Indeed, the study [12] provides a design process that ensures controllability, in a statefeedback setting.

- While the effort here has been focused on controllability, the results naturally translate to the dual question of observability from measurements of a subset of subsystems.

\section{REFERENCES}

[1] C. W. Wu and L. O. Chua, "Synchronization in an array of linearly coupled dynamical systems," IEEE Transactions on Circuits and Systems I: Fundamental Theory and Applications, vol. 42, no. 8, pp. 430-447, 1995.

[2] X. F. Wang and G. Chen, "Synchronization in scale-free dynamical networks: robustness and fragility," IEEE Transactions on Circuits and Systems I: Fundamental Theory and Applications, vol. 49, no. 1, pp. 54-62, 2002.

[3] L. M. Pecora and T. L. Carroll, "Master stability functions for synchronized coupled systems," Physical review letters, vol. 80, no. 10, p. 2109, 1998.

[4] R. Olfati-Saber, J. A. Fax, and R. M. Murray, "Consensus and cooperation in networked multi-agent systems," Proceedings of the IEEE, vol. 95, no. 1, pp. 215-233, 2007.

[5] T. Yang, S. Roy, Y. Wan, and A. Saberi, "Constructing consensus controllers for networks with identical general linear agents," International Journal of Robust and Nonlinear Control, vol. 21, no. 11, pp. $1237-$ 1256, 2011.

[6] Z. Li, W. Ren, X. Liu, and M. Fu, "Consensus of multi-agent systems with general linear and lipschitz nonlinear dynamics using distributed adaptive protocols," IEEE Transactions on Automatic Control, vol. 58, no. 7, pp. 1786-1791, 2013.

[7] Z. Li, Z. Duan, G. Chen, and L. Huang, "Consensus of multiagent systems and synchronization of complex networks: A unified viewpoint," IEEE Transactions on Circuits and Systems I: Regular Papers, vol. 57, no. 1, pp. 213-224, 2010.

[8] S. Zhang, M. Cao, and M. K. Camlibel, "Upper and lower bounds for controllable subspaces of networks of diffusively coupled agents," IEEE Transactions on Automatic control, vol. 59, no. 3, pp. 745-750, 2014.

[9] M. Xue and S. Roy, "Input-output properties of linearly-coupled dynamical systems: Interplay between local dynamics and network interactions," in Decision and Control, 2017 IEEE 56th Annual Conference on. IEEE, 2017, pp. 487-492.

[10] Y. Hao, Z. Duan, and G. Chen, "Further on the controllability of networked mimo lti systems," International Journal of Robust and Nonlinear Control, vol. 28, no. 5, pp. 1778-1788, 2018.

[11] L. Wang, G. Chen, X. Wang, and W. K. Tang, "Controllability of networked mimo systems," Automatica, vol. 69, pp. 405-409, 2016.

[12] Z. Ji, H. Lin, and H. Yu, "Protocols design and uncontrollable topologies construction for multi-agent networks," IEEE Transactions on Automatic Control, vol. 60, no. 3, pp. 781-786, 2015.

[13] N. Cai and Y.-S. Zhong, "Formation controllability of high-order linear time-invariant swarm systems," IET control theory \& applications, vol. 4, no. 4, pp. 646-654, 2010 .
[14] M. Xue and S. Roy, "Comment on "upper and lower bounds for controllable subspaces of networks of diffusively-coupled agents," IEEE Transactions on Automatic Control, 2017.

[15] R. A. Horn and C. R. Johnson, Matrix analysis. Cambridge university press, 1990.

[16] M. Xue and S. Roy, "Kronecker products of defective matrices: Some spectral properties and their implications on observability," in American Control Conference, 2012. IEEE, 2012, pp. 5202-5207.

[17] Z. Duan, G. Chen, and L. Huang, "Synchronization of weighted networks and complex synchronized regions," Physics Letters A, vol. 372, no. 21, pp. 3741-3751, 2008

[18] S.-H. Wang and E. Davison, "On the stabilization of decentralized control systems," IEEE Transactions on Automatic Control, vol. 18, no. 5, pp. 473-478, 1973.

[19] E. Davison and S. Wang, "A characterization of decentralized fixed modes in terms of transmission zeros," IEEE Transactions on Automatic Control, vol. 30, no. 1, pp. 81-82, 1985.

[20] B. D. Anderson and D. J. Clements, "Algebraic characterization of fixed modes in decentralized control," Automatica, vol. 17, no. 5, pp. 703-712, 1981.

[21] D. S. Watkins, The matrix eigenvalue problem: GR and Krylov subspace methods. Siam, 2007, vol. 101.

[22] L. Qiu and E. J. Davison, "The stability robustness of generalized eigenvalues," in Decision and Control, 1989., Proceedings of the 28th IEEE Conference on. IEEE, 1989, pp. 1902-1907.

[23] A. Rahmani, M. Ji, M. Mesbahi, and M. Egerstedt, "Controllability of multi-agent systems from a graph-theoretic perspective," SIAM Journal on Control and Optimization, vol. 48, no. 1, pp. 162-186, 2009. 